\title{
Nash Equilibria for competitive information diffusion on trees
}

\author{
Lucy Small $^{1}$, Oliver Mason ${ }^{*, 1}$ \\ Hamilton Institute, National University of Ireland Maynooth, Maynooth, Co. Kildare, Ireland
}

\section{A R T I C L E I N F O}

\section{Article history:}

Received 25 July 2012

Accepted 10 January 2013

Available online 16 January 2013

Communicated by J. Xu

\section{Keywords:}

Combinatorial problems

Competitive information diffusion

Game theory

Nash equilibrium

Trees

\begin{abstract}
A B S T R A C T
We consider the game theoretic model of competitive information diffusion recently introduced in Alon et al. (2010) [1]. We show that for the case of 2 competing agents, there exists a Nash Equilibrium for this game on any tree. We also present an example to show that this is not necessarily true for 3 or more agents.
\end{abstract}

(C) 2013 Elsevier B.V. All rights reserved.

\section{Introduction}

In the recent papers [1,2] a deterministic model for competitive information diffusion on social networks was introduced and studied. The model considers the diffusion process as a game played on the network by external agents. In contrast to many other game theoretic models for the diffusion of information and innovation [5,3], it considers competition between different innovations rather than the spread of a single innovation in a network. The main result of [1] claimed that any network of diameter 2 possesses a pure strategy Nash Equilibrium (N.E.). Unfortunately, as pointed out in [2], this result is not true without additional technical assumptions. In fact, even for the case of 2 competing agents on a network of diameter 2 , it is possible that the game introduced in [1] does not possess an N.E.

Rather than restricting the diameter of the network, we focus instead on hierarchical structures, which can be represented by a tree. We show that the model of [1] with 2

\footnotetext{
* Corresponding author. Tel.: +353 (0)1 7086274; fax: +353 5(0)1 7086269.

E-mail address: oliver.mason@nuim.ie (O. Mason).

1 Supported by the Irish Higher Educational Authority (HEA) PRTLI Network Mathematics Grant.
}

0020-0190/\$ - see front matter (C) 2013 Elsevier B.V. All rights reserved. http://dx.doi.org/10.1016/j.ipl.2013.01.011 competing agents always possesses an N.E. when the underlying graph is a tree. While the tree structure is clearly restrictive, it is worth noting that many social networks, including the online example twitter, are hierarchical in nature with 'leaders' and 'followers'. Moreover, our result makes no assumption concerning the diameter of the network; thus it opens an alternative line of research into the model of [1]. The layout of the note is as follows. In Section 2, we recall the basic model and set our notation. In Section 3, we present and prove our result, while in Section 4 we present our conclusions.

\section{Preliminaries}

All the graphs considered in this note are simple and undirected. For a graph $G$, we use $V(G)$ to denote its vertex set and $E(G)$ to denote its edge set. We denote an edge between $v, w$ in $V(G)$ by $v w$. For $v \in V(G)$, the neighbours of $v$ are given by $N(v)=\{w \in V(G) \mid v w \in E(G)\}$. If $v w \in E(G), G-v w$ denotes the graph obtained by removing the edge $v w$ from $E(G)$. Similarly if $v w \notin E(G)$, $G+v w$ denotes the graph obtained by adding the edge $v w$ to the edge set of $G$. Also for $v \in V(G), G-v$ denotes the graph obtained by removing $v$ and all of its incident edges from $G$. For a set $X,|X|$ denotes the cardinality of $X$.

A tree $T$ is a connected acyclic graph. It is well known that in a tree $T$, there exists a unique path between every 


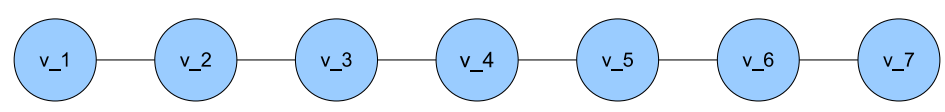

Fig. 1. Three agents and no Nash Equilibrium on $T$.

pair of distinct vertices $v, w$ in $V(T)$. Also, for every edge $v w \in E(T), T-v w$ is disconnected.

Our primary interest in the current paper is to study the game theoretic model of competitive information diffusion introduced in [1] on trees. We now recall the model of competitive information diffusion from [1].

\subsection{The game $\mathbf{D}$}

Let $G$ be a graph with $|V(G)|=N$ and a set of agents $[1, n]=\{1, \ldots, n\}$ be given. Initially $(t=0)$, each agent $i$ selects one vertex, $x_{i}$, in $V(G)$, which is labelled $i$. The $n$-tuple $\mathbf{x}=\left(x_{1}, \ldots, x_{n}\right)$ is known as a strategy profile. We only consider strategy profiles in which all of the $x_{i}$ are distinct. All other nodes are initially labelled 0 (corresponding to white nodes in [1]). We use -1 to denote grey nodes. In keeping with the original model of [1], grey nodes do not propagate. The basic paradigm is as follows. At time $t \geqslant 1$, if a white vertex $v$ (labelled 0 ) neighbours two vertices with distinct labels in $[1, n]$ at time $t-1$, then $v$ is labelled -1 (grey). If $v$ neighbours a vertex labelled $i \in[1, n]$ at time $t-1$ and has no neighbours labelled $j \in[1, n] \backslash\{i\}$, then $v$ is labelled $i$. Otherwise, $v$ 's label is unchanged.

Throughout the note, we refer to the above process as the game $\mathbf{D}$. The process terminates in a finite number of steps when no further vertices can be labelled $i \in[1, n]$. The utility $U_{i}(\mathbf{x})$ of agent $i$ is the total number of vertices labelled $i$ when the process terminates. In a slight abuse of notation, we shall occasionally write $U_{i}\left(x_{1}, \ldots, x_{n}\right)$ for $U_{i}(\mathbf{x})$. A strategy profile $\mathbf{x}=\left(x_{1}, \ldots, x_{n}\right)$ is a Nash Equilibrium (N.E.) for $\mathbf{D}$ if no agent can unilaterally improve their utility by changing to another starting vertex. Formally if

$U_{i}\left(x_{1}, \ldots, x_{n}\right) \geqslant U_{i}\left(x_{1}, \ldots, x_{i-1}, v, x_{i+1}, \ldots, x_{n}\right)$

for all $v \in V(G) \backslash\left\{x_{1}, \ldots, x_{n}\right\}$.

\section{Main result}

In this section, we show that for 2 agents competing on a tree, there always exists a Nash Equilibrium (N.E.) for the game D. Moreover, our result characterises the N.E. on a tree $T$. We first note that for the general game with $n>2$ agents, this conclusion does not necessarily hold.

Example 3.1. Consider the game D on the tree in Fig. 1 with 7 vertices and 3 agents.

Consider, without loss of generality, a strategy profile $\mathbf{x}=\left(v_{i}, v_{j}, v_{k}\right)$ where $i<j<k$.

If $v_{i} v_{j} \notin E(T)$ then $U_{1}(\mathbf{x})$ is increased by player 1 switching to vertex $v_{j-1}$. If $v_{j} v_{k} \notin E(T), U_{3}(\mathbf{x})$ is increased by player 3 choosing vertex $v_{j+1}$.

If $v_{i} v_{j} \in E(T)$ and $v_{j} v_{k} \in E(T)$ then $U_{2}(\mathbf{x})=1$, and it is always possible for player 2 to improve their utility by moving to either $v_{i-1}$ or $v_{k+1}$. Thus there is no N.E. for D on $T$.
For the remainder of this section, we consider the game D with $n=2$ agents on a tree $T$ with vertex set $V$ and edge set $E$.

The next lemma notes that once one agent selects an initial vertex $v$, the optimal choice for the other agent is a neighbour of $v$.

Lemma 3.1. Let $v \in V$ be given. There exists $w \in N(v)$ such that

$U_{2}(v, w)=\max _{x \in V} U_{2}(v, x)$.

Proof. Suppose the degree of $v$ is $d$. As $T$ is a tree, it is readily seen that $T-v$ has $d$ connected components $C_{1}, \ldots, C_{d}$. Let $u_{i}$ denote the neighbour of $v$ that is in $C_{i}$ for $1 \leqslant i \leqslant d$.

If $x \in V\left(C_{i}\right), y \in V\left(C_{j}\right)$, with $i \neq j$, the unique path in $T$ from $x$ to $y$ includes $v$. Hence, agent 2 cannot colour vertices in more than one component of $T-v$. Thus $\max _{x \in V} U_{2}(v, x)=\max _{1 \leqslant i \leqslant d}\left|V\left(C_{i}\right)\right|$.

Let $k$ be such that $\left|V\left(C_{k}\right)\right|=\max _{1 \leqslant i \leqslant d}\left|V\left(C_{i}\right)\right|$. Choose $w \in N(v) \cap V\left(C_{k}\right)$. For any $u \in V\left(C_{k}\right)$ the unique path (in $T$ ) from $v$ to $u$ must contain $w$. It follows that all vertices in $C_{k}$ are labelled 2. Hence,

$U_{2}(v, w)=\left|V\left(C_{k}\right)\right|=\max _{x \in V} U_{2}(v, x)$

as claimed.

Lemma 3.2. Let $\mathbf{x}=(v, w)$ be a strategy profile such that $v w \in$ $E(T)$. Let $u \in N(v) \backslash\{w\}$ and consider the strategy profile $\mathbf{x}^{\prime}=$ $(v, u)$. Then $U_{2}\left(\mathbf{x}^{\prime}\right)<U_{1}(\mathbf{x})$.

Proof. As $T$ is a tree, $T-v w$ has two connected components. Write $C_{v}, C_{w}$ for the component of $T-v w$ containing $v, w$ respectively. It is clear that all vertices in $C_{v}$ will be labelled 1 and all the vertices in $C_{w}$ are labelled 2 . Hence, $U_{1}(\mathbf{x})=\left|V\left(C_{v}\right)\right|$.

Now consider the profile $\mathbf{x}^{\prime}=(v, u)$ where $u \in$ $N(v) \backslash\{w\}$. Then $u \in V\left(C_{v}\right)$. Hence, for the strategy profile $\mathbf{x}^{\prime}$, no vertices in $C_{w}$ are labelled 2 , since the path to any $c \in V\left(C_{w}\right)$ must contain $v$. It follows that the vertices labelled 2 form a subset of $V\left(C_{v}\right) \backslash\{v\}$. Thus

$U_{2}\left(\mathbf{x}^{\prime}\right) \leqslant\left|V\left(C_{v}\right)\right|-1<U_{1}(\mathbf{x})$.

The following lemma shows that if a strategy profile consists of two neighbouring vertices, then all vertices are either labelled 1 or 2 when the process terminates.

Lemma 3.3. Consider a strategy profile $\mathbf{x}=(v, w)$ such that $v w \in E(T)$. Then $U_{1}(\mathbf{x})+U_{2}(\mathbf{x})=|V(T)|$.

Proof. As $v, w$ are neighbours, they have no common neighbour as $T$ is a tree. Further, $T-v w$ consists of two connected components, $C_{v}$ and $C_{w}$. By similar arguments 
to those employed in the previous results, it can be seen that every vertex in $C_{v}$ is labelled 1 while every vertex in $C_{w}$ is labelled 2. So $U_{1}(\mathbf{x})=\left|V\left(C_{v}\right)\right|, U_{2}(\mathbf{x})=\left|V\left(C_{w}\right)\right|$ and hence $U_{1}(\mathbf{x})+U_{2}(\mathbf{x})=|V(T)|$.

We can now state the main result of the note, which shows that the game D with 2 agents has an N.E. on any tree of any diameter.

Theorem 3.1. Let $\mathbf{X}=(v, w)$ be a strategy profile on a tree $T$ such that $v w \in E(T)$ and

$$
\left|U_{1}(v, w)-U_{2}(v, w)\right|=\min _{y z \in E(T)}\left|U_{1}(y, z)-U_{2}(y, z)\right| .
$$

Then $\mathbf{x}$ is a Nash Equilibrium.

Proof. Without loss of generality, assume $U_{2}(\mathbf{x}) \geqslant U_{1}(\mathbf{x})$. By Lemma 3.1, we know that there is some $u \in N(v)$ such that

$U_{2}(v, u) \geqslant U_{2}(v, y)$

for all $y \in V(T)$. However, it follows from Lemma 3.2 that

$U_{2}(v, u) \leqslant U_{1}(v, w) \leqslant U_{2}(v, w)$.

Thus agent 2 certainly cannot increase their utility by unilaterally changing strategy.

Next suppose that there exists some $u \in V(T)$ such that $U_{1}(u, w)>U_{1}(v, w)$. Lemma 3.1 implies that there exists such a $u$ in $N(w)$. From Lemma 3.3 we know that

$U_{1}(u, w)+U_{2}(u, w)=|V(T)|=U_{1}(v, w)+U_{2}(v, w)$.

It follows immediately that $U_{2}(u, w)<U_{2}(v, w)$. We now show that this leads to a contradiction. There are three cases to consider.

Case 1: $U_{1}(u, w) \geqslant U_{2}(v, w)$

From Lemma 3.2, we know that $U_{1}(u, w) \leqslant U_{2}(v, w)-$ 1 so this cannot happen.

Case 2: $U_{1}(u, w)<U_{2}(v, w)$, and $U_{2}(u, w)>U_{1}(u, w)$

We know that $U_{2}(v, w)>U_{2}(u, w)$, and $U_{1}(u, w)>$

$U_{1}(v, w)$. Thus, $U_{2}(v, w)+U_{1}(u, w)>U_{1}(v, w)+U_{2}(u, w)$. Rearranging, we see that

$U_{2}(v, w)-U_{1}(v, w)>U_{2}(u, w)-U_{1}(u, w)>0$

which contradicts our initial assumption, that $\mid U_{2}(v, w)-$ $U_{1}(v, w) \mid$ is minimal.

Case 3: $U_{1}(u, w)<U_{2}(v, w)$, and $U_{2}(u, w) \leqslant U_{1}(u, w)$ We know from Lemma 3.2 that $U_{1}(u, w) \leqslant U_{2}(v, w)-1$. It similarly follows that $U_{2}(u, w) \geqslant U_{1}(v, w)+1$. Taken

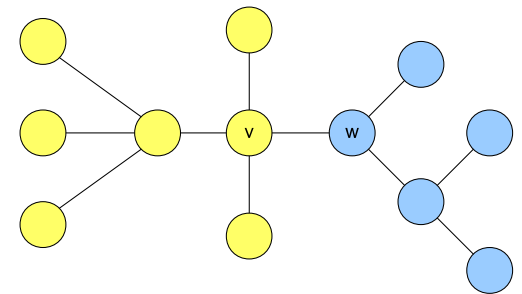

Fig. 2. The tree $T$.

together, these observations imply that

$$
\begin{aligned}
\left|U_{1}(u, w)-U_{2}(u, w)\right| & =U_{1}(u, w)-U_{2}(u, w) \\
& \leqslant U_{2}(v, w)-U_{1}(v, w)-2 \\
& <U_{2}(v, w)-U_{1}(v, w) .
\end{aligned}
$$

This is again a contradiction.

Putting the above arguments together, we see that $\mathbf{x}=$ $(v, w)$ is an N.E. as claimed.

Example 3.2. In the tree $T$ in Fig. 2 there is a unique Nash Equilibrium, $\mathbf{x}=(v, w) .\left|U_{1}(\mathbf{x})-U_{2}(\mathbf{x})\right|=|7-5|=2$ is clearly minimal over all pairs of neighbours.

\section{Concluding remarks}

As highlighted by the work of $[1,2]$ and $[3,4]$, identifying conditions for the existence of N.E. for games on graphs is a difficult problem. We have shown that the model of competitive information diffusion studied in [1,2] always admits an N.E. on a tree when the number of agents is 2 . While trees are a restrictive class of graphs, they can serve as an idealisation of hierarchical structures that arise in many social networks. Identifying other structures which guarantee the existence of an N.E. and characterising these when they exist remains a challenging question for future research.

\section{References}

[1] N. Alon, M. Feldman, A. Procaccia, M. Tennenholtz, A note on competitive information diffusion through social networks, Information Processing Letters 110 (2010) 221-225.

[2] R. Takehara, M. Hachimori, M. Shigeno, A comment on pure-strategy Nash equilibria in competitive diffusion games, Information Processing Letters 112 (2012) 59-60.

[3] J.R. Davis, et al., Equilibria and efficiency loss in games on networks, Internet Mathematics 7 (3) (2011) 178-205.

[4] C. Dürr, N.K. Thang, Nash equilibria in Voronoi games on graphs, in: European Symposium on Algorithms, 2007.

[5] A. Galeotti, S. Goyal, M. Jackson, F. Vega-Redondo, L. Yariv, Network games, The Review of Economic Studies 77 (2010) 218-244. 